\title{
On bases from cosines in Lebesgue spaces with variable summability index
}

\author{
Togrul Muradov ${ }^{1 *}$ and Chingiz Hashimov ${ }^{1,2}$
}

\section{"Correspondence:}

togrulmuradov@gmail.com

'Department of Non-Harmonic

Analysis, Institute of Mathematics

and Mechanics of NAS of

Azerbaijan, 9 B. Vahabzadeh Str.,

Baku, 1141, Azerbaijan

Full list of author information is

available at the end of the article

\begin{abstract}
In this paper the perturbed system of cosines is considered. Under certain conditions on the summability index $p(\cdot)$ and perturbation, the basicity of this system in Lebesgue spaces $L_{p(\cdot)}(0, \pi)$ with variable summability index $p(\cdot)$ is proved. The obtained results generalize similar results for the case $p(\cdot)=p=$ const.
\end{abstract}

Keywords: basicity; perturbation; system of cosines; variable exponent

\section{Introduction}

Perturbed systems of exponents, cosines and sines play an important role in the theory of spectral theory of differential operators, in the theory of optimal control, in approximation theory and so on. Therefore there are a lot of papers studying the frame properties and also the basis properties (completeness, minimality, basicity, etc.) of the perturbed trigonometric systems in various Banach spaces of functions. For more detailed information see [1-12].

In connection with applications in mechanics and theoretical physics in recent years there is a great interest in studying different problems in Lebesgue spaces with variable summability index. For many results in this direction one can see [13] and also [14, 15]. Basis properties of some trigonometric systems and other systems of functions (Haar system, classical system of Legendre, etc.) were investigated [6, 7, 16, 17].

In this paper a perturbed system of cosines is considered. Stability of the basicity of this system in Lebesgue space with variable summability index is studied. It should be noted that the basicity in generalized Lebesgue space of perturbed systems of exponents was considered earlier in $[16,17]$.

\section{Necessary information}

A Banach space will be called a $B$-space. A Banach space of sequences of scalars over the field $K$ will be called a $K$-space. We give some information on Lebesgue spaces with variable summability index.

Let $p:[-\pi, \pi] \rightarrow[1,+\infty)$ be some Lebesgue measurable function. Denote the class of all functions measurable on $[-\pi, \pi]$ (with respect to the Lebesgue measure) by $\mathscr{L}_{0}$.

Let us choose the notation

$$
I_{p}(f) \stackrel{\text { def }}{=} \int_{-\pi}^{\pi}|f(t)|^{p(t)} d t
$$

(0) 2016 Muradov and Hashimov. This article is distributed under the terms of the Creative Commons Attribution 4.0 International License (http://creativecommons.org/licenses/by/4.0/), which permits unrestricted use, distribution, and reproduction in any medium, provided you give appropriate credit to the original author(s) and the source, provide a link to the Creative Commons license, and indicate if changes were made. 
Let

$$
\mathscr{L} \equiv\left\{f \in \mathscr{L}_{0}: I_{p}(f)<+\infty\right\}
$$

With respect to ordinary linear operations of addition of functions and multiplication by a number, for

$$
p^{+}=\sup \underset{[-\pi, \pi]}{\operatorname{vrai}} p(t)<+\infty
$$

$\mathscr{L}$ turns into a linear space. With the norm

$$
\|f\|_{p(\cdot)} \stackrel{\text { def }}{=} \inf \left\{\lambda>0: I_{p}\left(\frac{f}{\lambda}\right) \leq 1\right\}
$$

$\mathscr{L}$ is a Banach space and we denote it by $L_{p(.)}$. Assume

$$
W L_{0} \stackrel{\text { def }}{=}\left\{p: \exists C>0, \forall t_{1}, t_{2} \in[0, \pi]:\left|t_{1}-t_{2}\right| \leq \frac{1}{2} \Rightarrow\left|p\left(t_{1}\right)-p\left(t_{2}\right)\right| \leq \frac{C}{-\ln \left|t_{1}-t_{2}\right|}\right\} .
$$

Everywhere $q(\cdot)$ denotes the function conjugate to $p(\cdot): \frac{1}{p(t)}+\frac{1}{q(t)} \equiv 1$. Choose

$$
p^{-}=\inf \underset{[-\pi, \pi]}{\operatorname{vrai}} p(t)
$$

Hölder's generalized inequality holds:

$$
\int_{-\pi}^{\pi}|f(t) g(t)| d t \leq c\left(p^{-} ; p^{+}\right)\|f\|_{p(\cdot)}\|g\|_{q(\cdot)}
$$

where $c\left(p^{-} ; p^{+}\right)=1+\frac{1}{p^{-}}-\frac{1}{p^{+}}$.

The following property that we will use is obvious.

Property A If $|f(t)| \leq|g(t)|$ a.e. on $(-\pi, \pi)$, then $\|f\|_{p_{(\cdot)}} \leq\|g\|_{p_{(\cdot)}}$.

For detailed information on the space $L_{p(\cdot)}$ one can see [14,15] and also [13].

We will also use the notion of the space of coefficients. Let us define it. Let $\vec{x} \equiv\left\{x_{n}\right\}_{n \in N} \subset$ $X$ be a non-degenerate system in $B$-space $X$, i.e. $x_{n} \neq 0, \forall n \in N$.

Assume

$$
\mathscr{K}_{\breve{x}} \equiv\left\{\left\{\lambda_{n}\right\}_{n \in N} \text { : the series } \sum_{n=1}^{\infty} \lambda_{n} x_{n} \text { converges in } X\right\} .
$$

Introduce the norm in $\mathscr{K}_{\vec{x}}:$

$$
\|\vec{\lambda}\|_{\mathscr{K}_{\bar{x}}}=\sup _{m}\left\|\sum_{n=1}^{m} \lambda_{n} x_{n}\right\|, \quad \text { where } \vec{\lambda}=\left\{\lambda_{n}\right\}_{n \in N} .
$$

With respect to ordinary operations of addition and multiplication by a complex number, $\mathscr{K}_{\hat{x}}$ is a Banach space. We also need some notion and facts from the basis theory. 
Definition 1 Let $X$ be some $B$-space. We call the system $\left\{\varphi_{n}\right\}_{n \in N} \subset X \omega$-linear independent in $X$ (or simply $\omega$-linear independent) if from $\sum_{n} c_{n} \varphi_{n}=0$ it follows $c_{n}=0, \forall n \in N$.

The following theorem is valid.

Theorem 1 Let $X$ be B-space with the basis $\left\{\varphi_{n}\right\}_{n \in N}$ and $F: X \rightarrow X$ be a Fredholmoperator. Then for the system $\left\{\psi_{n}\right\}_{n \in N}$ where $\psi_{n}=F \varphi_{n}, \forall n \in N$, the following properties in $X$ are equivalent:

(a) $\left\{\psi_{n}\right\}_{n \in N}$ is complete in $X$;

(b) $\left\{\psi_{n}\right\}_{n \in N}$ is minimal in $X$;

(c) $\left\{\psi_{n}\right\}_{n \in N}$ is $\omega$-linear independent in $X$;

(d) $\left\{\psi_{n}\right\}_{n \in N}$ is a bases isomorphic to $\left\{\varphi_{n}\right\}_{n \in N}$ in $X$.

From this theorem we have the following.

Corollary 1 Let $\left\{\varphi_{n}\right\}_{n \in N}$ form a basis for $X$ and card $\left\{n: \psi_{n} \neq \varphi_{n}\right\}<+\infty$. Then with respect to the system $\left\{\psi_{n}\right\}_{n \in N}$ the statement of Theorem 1 is valid.

For these or other results one can see for example [1-5].

Definition 2 We call the system $\left\{\varphi_{n}\right\}_{n \in Z_{+}} \subset L_{p(\cdot)} \mathscr{K}$-Hilbert if $\exists \delta>0$ :

$$
\delta\left\|\left\{c_{n}\right\}\right\|_{\mathscr{K}} \leq\left\|\sum c_{n} \varphi_{n}\right\|_{L_{p(\cdot)}},
$$

for an arbitrary finite set $\left\{c_{n}\right\}$.

We will also use the following.

Definition 3 The sequence $\left\{\lambda_{n}\right\}_{n \in N}$ is called separated, if $\inf _{i \neq j}\left|\lambda_{i}-\lambda_{j}\right|>0$.

For more details regarding these and other results one can see [1-5].

\section{Basic results}

Let $\mathscr{K}$ be some $K$-space with the norm $\|\cdot\|_{\mathscr{K}}$. Assume that the norm $\|\cdot\|_{\mathscr{K}}$ satisfies the following condition:

( $\alpha)\left\|\left\{\lambda_{k}\right\}_{k \in N}\right\|_{\mathscr{K}}=\left\|\left\{\lambda_{\pi(k)}\right\}_{k \in N}\right\|_{\mathscr{K}}$ for an arbitrary permutation $\pi: N \rightarrow N$.

Assume that the system $\left\{\cos \lambda_{n} x\right\}_{n \in N}$ is $\mathscr{K}$-Hilbert in $L_{p(\cdot)}$, where $\left\{\lambda_{n}\right\}_{n \in N} \in \mathscr{K}$ is some sequence. Taking into account the inequality

$$
\left|\cos \lambda_{n} x-\cos \lambda_{k} x\right| \leq \pi\left|\lambda_{n}-\lambda_{k}\right|, \quad \forall x \in[0, \pi]
$$

from condition $(\alpha)$ and Property A we immediately get

$$
\begin{aligned}
0 & <\delta\|\{1 ; 1 ; 0 ; \ldots\}\|_{\mathscr{K}} \leq \delta\left\|\cos \lambda_{n} x-\cos \lambda_{k} x\right\|_{L_{p(\cdot)}} \\
& \leq \delta \pi\|1\|_{L_{p(\cdot)}}\left|\lambda_{n}-\lambda_{k}\right|, \quad \forall n, k \in N .
\end{aligned}
$$

Thus, the following lemma is valid. 
Lemma 1 Let the $K$-space $\mathscr{K}$ satisfy condition $(\alpha)$ and the system of cosines $\left\{\cos \lambda_{n} x\right\}_{n \in N}$ be a $\mathscr{K}$-Hilbert in $L_{p(\cdot)}$ Then the sequence $\left\{\lambda_{n}\right\}_{n \in N}$ is separated.

The analog of Levinson's well-known theorem [18] on the completeness of the system of exponents is valid in this case as well.

Theorem 2 Let $1<p^{-} \leq p^{+}<+\infty$. If from the system of functions $\left\{e^{i \lambda_{k} x}\right\}$ complete in $L_{p(\cdot)}(-\pi, \pi)$ we reject $n$ arbitrary functions and instead of them add other functions $e^{i \mu_{j} x}$, $j=\overline{1, n}$, wherein $\mu_{k}, k=\overline{1, n}$, are arbitrary pairwise different complex numbers not equal to any of the numbers $\lambda_{k}$, then the new system will also be complete in $L_{p(\cdot)}(-\pi, \pi)$.

The proof of this theorem is conducted by analogy with the case $L_{p}(-\pi, \pi)(i . e . p(x) \equiv p=$ const). As under the conditions of the theorem $\left(L_{p(\cdot)}(-\pi, \pi)\right)^{*}=L_{q(\cdot)}(-\pi, \pi), \frac{1}{p(x)}+\frac{1}{q(x)}=1$. The following theorem is also valid.

Theorem 3 Let $\left\{\lambda_{n}\right\}_{n \in N} \subset C$ be an arbitrary sequence of various numbers, and $1<p^{-} \leq$ $p^{+}<+\infty$. The system $\left\{\cos \lambda_{n} x\right\}_{n \in N}$ is complete in $L_{p(\cdot)}(0, \pi)$ if and only if the system $\left\{e^{ \pm i \lambda_{n} x}\right\}_{n \in N}$ is complete in $L_{p(\cdot)}(-\pi, \pi)$. If for some $k_{0}: \lambda_{k_{0}}=0$, then instead of the functions $e^{i \lambda_{k_{0}} x}$ and $e^{-i \lambda_{k_{0}} x}$ the functions 1 and $x$ should be taken.

From these two theorems we immediately have the following.

Corollary 2 Let $1<p^{-} \leq p^{+}<+\infty$. If from the system of functions $\left\{\cos \lambda_{k} x\right\}$ complete in $L_{p(\cdot)}(0, \pi)$ we reject $n$ arbitrary functions and instead of them add other $n$ functions $\left\{\cos \mu_{j} x\right\}, j=\overline{1, n}$, wherein $\mu_{k}, k=\overline{1, n}$, are arbitrary complex numbers such that $\mu_{i} \neq \pm \mu_{j}$ for $i \neq j$, and not equal to any of the numbers $\pm \lambda_{k}$, then the obtained system will also be complete in $L_{p(\cdot)}(0, \pi)$.

Now we cite the basic result of the paper.

Theorem 4 Let $1<p^{-} \leq p^{+}<+\infty$ and $\left\{\lambda_{n} ; \mu_{n}\right\}_{n \in Z_{+}} \subset R$ be some sequence of different numbers such that for some $\alpha \in\left(1, p_{0}\right]$

$$
\sum_{n=0}^{\infty}\left|\lambda_{n}-\mu_{n}\right|^{\alpha}<+\infty
$$

where $p_{0}=\min \left\{2 ; p^{-}\right\}$. If the system $\left\{\cos \lambda_{n} x\right\}_{n \in Z_{+}}$forms a basis for $L_{p(\cdot)}(0, \pi)$ equivalent to the basis $\{\cos n x\}_{n \in Z_{+}}$, then the system $\left\{\cos \mu_{n} x\right\}_{n \in Z_{+}}$also forms a basis for $L_{p(\cdot)}(0, \pi)$ equivalent to the basis $\{\cos n x\}_{n \in Z_{+}}$.

Proof We have

$$
\left|\cos \lambda_{n} x-\cos \mu_{n} x\right| \leq \pi\left|\lambda_{n}-\mu_{n}\right|, \quad n \in Z_{+} .
$$

Consequently

$$
\left\|\cos \lambda_{n} x-\cos \mu_{n} x\right\|_{p(\cdot)} \leq c\left|\lambda_{n}-\mu_{n}\right|, \quad n \in Z_{+},
$$


where $c$ means a constant (which later may be different at different places) dependent only on $p(\cdot)$. As a result we get

$$
\sum_{n=0}^{\infty}\left\|\cos \lambda_{n} x-\cos \mu_{n} x\right\|_{p(\cdot)}^{\alpha} \leq c \sum_{n=0}^{\infty}\left|\lambda_{n}-\mu_{n}\right|^{\alpha}<+\infty
$$

We denote the space of coefficients of the system $\left\{\varphi_{n}\right\}_{n \in N} \subset L_{p(\cdot)}(0, \pi)$ by $\mathscr{K}\left(\left\{\varphi_{n}\right\}_{n \in N}\right)$.

Thus, by the conditions of the theorem we have

$$
\mathscr{K}_{p(\cdot)} \equiv \mathscr{K}\left(\left\{\cos \lambda_{n} x\right\}_{n \in Z_{+}}\right) \equiv \mathscr{K}\left(\{\cos n x\}_{n \in Z_{+}}\right) .
$$

Therefore it is obvious that $\exists M>0$ :

$$
\begin{aligned}
M^{-1}\left\|\sum_{n=0}^{\infty} c_{n} \cos \lambda_{n} x\right\|_{p(\cdot)} & \leq\left\|\sum_{n=0}^{\infty} c_{n} \cos n x\right\|_{p(\cdot)} \\
& \leq M\left\|\sum_{n=0}^{\infty} c_{n} \cos \lambda_{n} x\right\|_{p(\cdot)}, \quad \forall\left\{c_{n}\right\}_{n \in Z_{+}} \in \mathscr{K}_{p(\cdot) .}
\end{aligned}
$$

Assume $\frac{1}{\alpha}+\frac{1}{\beta}=1$. It is clear that $\beta \geq 2$. The following continuous embeddings hold:

$$
L_{p(\cdot)}(0, \pi) \subset L_{p^{-}}(0, \pi) \subset L_{\alpha}(0, \pi) .
$$

From the classic Hausdorff-Young theorem it follows that

$$
\left(\sum_{n=0}^{\infty}\left|c_{n}\right|^{\beta}\right)^{1 / \beta} \leq M_{\alpha}\left\|\sum_{n=0}^{\infty} c_{n} \cos n x\right\|_{L_{\alpha}(0, \pi)}, \quad \forall\left\{c_{n}\right\}_{n \in Z_{+}} \in l_{\beta}^{0},
$$

where

$$
l_{\beta}^{0} \equiv\left\{\left\{c_{n}\right\}_{n \in Z_{+}}: \exists n_{0} \geq 0 \Rightarrow c_{k}=0, \forall k \geq n_{0}+1\right\},
$$

and $M_{\alpha}$ is a constant dependent only on $\alpha$. Let $\varepsilon>0$ be an arbitrary number. Then it is clear that $\exists n_{\varepsilon} \in Z_{+}$:

$$
\sum_{n=n_{\varepsilon}}^{\infty}\left\|\cos \lambda_{n} x-\cos \mu_{n} x\right\|_{p(\cdot)}^{\alpha}<\varepsilon
$$

Assume

$$
\varphi_{n}(x)= \begin{cases}\cos \lambda_{n} x, & n=\overline{0, n_{\varepsilon}-1} \\ \cos \mu_{n} x, & n \geq n_{\varepsilon}, n \in Z_{+} .\end{cases}
$$

Take $\forall\left\{c_{n}\right\}_{n \in Z_{+}} \in l_{\beta}^{0}$. We have

$$
\begin{aligned}
\left\|\sum_{n=0}^{\infty} c_{n}\left(\varphi_{n}(x)-\cos \lambda_{n} x\right)\right\|_{p(\cdot)} & \leq \sum_{n=0}^{\infty}\left|c_{n}\right|\left\|\varphi_{n}(x)-\cos \lambda_{n} x\right\|_{p(\cdot)} \\
& \leq\left(\sum_{n=0}^{\infty}\left|c_{n}\right|^{\beta}\right)^{1 / \beta}\left(\sum_{n=0}^{\infty}\left\|\varphi_{n}(x)-\cos \lambda_{n} x\right\|_{p(\cdot)}^{\alpha}\right)^{1 / \alpha} .
\end{aligned}
$$


Taking into account equations (3) and (4), we get

$$
\begin{aligned}
& \left\|\sum_{n=0}^{\infty} c_{n}\left(\varphi_{n}(x)-\cos \lambda_{n} x\right)\right\|_{p(\cdot)} \\
& \quad \leq M_{\alpha}\left\|\sum_{n=0}^{\infty} c_{n} \cos n x\right\|_{L_{\alpha}(0, \pi)}\left(\sum_{n=n_{\varepsilon}}^{\infty}\left\|\cos \lambda_{n} x-\cos \mu_{n} x\right\|_{p(\cdot)}^{\alpha}\right)^{1 / \alpha} \\
& \quad \leq M_{\alpha} \varepsilon^{\frac{1}{\alpha}}\left\|\sum_{n=0}^{\infty} c_{n} \cos n x\right\|_{L_{\alpha}(0, \pi)} .
\end{aligned}
$$

Having paid attention to the embedding (2) and equation (1), we finally get

$$
\left\|\sum_{n=0}^{\infty} c_{n}\left(\varphi_{n}(x)-\cos \lambda_{n} x\right)\right\|_{p(\cdot)} \leq M M_{\alpha} \varepsilon^{\frac{1}{\alpha}}\left\|\sum_{n=0}^{\infty} c_{n} \cos \lambda_{n} x\right\|_{p(\cdot)} .
$$

Take $\varepsilon$ so small that the inequality $m_{\alpha}=M M_{\alpha} \varepsilon^{\frac{1}{\alpha}}<1$ is fulfilled. Then by the Paley-Wiener theorem and from inequalities (5) it follows that the system $\left\{\varphi_{n}(\cdot)\right\}_{n \in Z_{+}}$forms a basis for $L_{p(\cdot)}(0, \pi)$ equivalent to the basis $\left\{\cos \lambda_{n} x\right\}_{n \in Z_{+}}$. Then we replace in the system $\left\{\varphi_{n}\right\}_{n \in Z_{+}}$ the elements $\left\{\varphi_{0} ; \ldots ; \varphi_{n_{\varepsilon}-1}\right\}$ by $\left\{\cos \mu_{0} x ; \ldots ; \cos \mu_{n_{\varepsilon}-1}\right\}$. From Corollary 2 it follows that the system $\left\{\cos \mu_{n} x\right\}_{n \in Z_{+}}$is complete in $L_{p(\cdot)}(0, \pi)$. Then, as follows from Corollary 1 , it forms a basis for $L_{p(\cdot)}(0, \pi)$ equivalent to the basis $\left\{\cos \lambda_{n} x\right\}_{n \in Z_{+}}$and as a result is equivalent to the basis $\{\cos n x\}_{n \in Z_{+}}$. The theorem is proved. The validity of the following theorem is established in the same way.

Theorem 5 Let with respect to the sequences $\left\{\lambda_{n}\right\}_{n \in N}$ and $\left\{\mu_{n}\right\}_{n \in N}$ all the conditions of Theorem 4 be fulfilled. If the system of sines $\left\{\sin \lambda_{n} x\right\}_{n \in N}$ forms a basis for $L_{p(\cdot)}(0, \pi)$ equivalent to the basis $\{\sin n x\}_{n \in N}$, then the system $\left\{\sin \mu_{n} x\right\}_{n \in N}$ also forms a basis for $L_{p(\cdot)}(0, \pi)$ equivalent to the basis $\{\sin n x\}_{n \in N}$.

\section{Example}

The following example is of independent interest. Let us consider the following Cauchy problem :

$$
\left.\begin{array}{l}
-y^{\prime \prime}(x)+q(x) y(x)=\lambda^{2} y(x), \quad x \in(0, \pi), \\
y(0)=1, \quad y^{\prime}(0)=\sigma,
\end{array}\right\}
$$

where $q(x) \in L_{1}(0, \pi)$ is a real function, $\sigma \in R$. One can understand this problem in the sense of Il'in [19]. The following question is interesting: for what sequences $\left\{\lambda_{n}\right\}_{n \in N} \subset R$ does the system $\left\{y_{\lambda_{n}}(x)\right\}_{n \in N}$ being the solution of problem (6) form a basis for $L_{p(\cdot)}(0, \pi)$ ? We have the following relation:

$$
y_{\lambda}(x)=\cos \lambda x+\int_{0}^{x} K(x, t) \cos \lambda t d t,
$$

where $K(x, t)$ is a continuous on $[0, \pi]$ function.

With respect to this fact refer to [20]. 
Denote by $K$ an operator determined by the expression

$$
[K f](x)=\int_{0}^{x} K(x, t) f(t) d t .
$$

It is obvious that $K$ is Volterrian and so the operator $I+K$ is boundedly invertible in $L_{p(\cdot)}$ $\left(I \in L\left(L_{p(\cdot)} ; L_{p(\cdot)}\right)\right.$ is a unit operator). Then from the relation $y_{\lambda}(x)=(I+K) \cos \lambda x$ and from the results of the previous item we see that the system $\left\{y_{\lambda_{n}}(x)\right\}_{n \in N}$ is a basis in $L_{p(\cdot)}$ only if the system of $\operatorname{cosines}\left\{\cos \lambda_{n} x\right\}_{n \in N}$ forms a basis for $L_{p(\cdot)}(0, \pi)$.

Thus, the following theorem is valid.

Theorem 6 Let $p(\cdot) \in W L_{0}, p^{-}>1, q \in L_{1}$, and the sequence $\left\{\lambda_{n}\right\}_{n \in N} \subset R$ satisfy the condition $\sum_{n=0}^{\infty}\left|\lambda_{n}-n\right|^{\alpha}<+\infty$, where $\alpha=\min \left\{2 ; p^{-}\right\}$. Then the system $\left\{y_{\lambda_{n}}(x)\right\}_{n \in N}$ from the solution of the Cauchy problem (6) is a basis in $L_{p(\cdot)}$, equivalent to the basis $\{\cos n x\}_{n \in Z_{+}}$in $L_{p(\cdot)}(0, \pi)$.

\section{Competing interests}

The authors declare that they have no competing interests.

\section{Authors' contributions}

The authors contributed equally to this work. All authors read and approved the final manuscript.

\section{Author details}

'Department of Non-Harmonic Analysis, Institute of Mathematics and Mechanics of NAS of Azerbaijan, 9 B. Vahabzadeh Str., Baku, 1141, Azerbaijan. ${ }^{2}$ Ganja State University, Ganja, Azerbaijan.

\section{Acknowledgements}

This work was supported by the Research Program Competition launched by the National Academy of Sciences of Azerbaijan (Program: Frame theory Applications of Wavelet Analysis to Signal Processing in Seismology and Other Fields). The authors express their deep gratitude to Professor BT Bilalov, corresponding member of the National Academy of Sciences of Azerbaijan, for his inspiring guidance and valuable suggestions during the work.

Received: 9 July 2015 Accepted: 21 December 2015 Published online: 04 January 2016

\section{References}

1. Young, RM: An Introduction to Nonharmonic Fourier Series. Springer, Berlin (1980) 246 pp.

2. Singer, l: Bases in Banach Spaces, vol. 1. Springer, Berlin (1970) 673 pp.

3. Heil, C: A Basis Theory Primer. Springer, Berlin (2011) 536 pp.

4. Christensen, O: An Introduction to Frames and Riesz Bases Birkhäuser Boston, Boston (2003) xxii+440 pp.

5. Bilalov, BT: Bases of exponents cosines and sines being the eigenfunctions of differential operators. Differ. Uravn. 39(5), 1-5 (2003)

6. Sharapudinov, II: Some problems in approximation theory in the spaces Lp(x)(E). Anal. Math. 33(2), 135-153 (2007)

7. Bilalov, BT, Guseynov, ZG: Basicity of a system of exponents with a piece-wise linear phase in variable spaces. Mediterr. J. Math. 9(3), 487-498 (2012)

8. He, X, Volkmer, H: Riesz bases of solutions of Sturm-Liouville equations. J. Fourier Anal. Appl. 7(3), $297-307$ (2001)

9. Russell, DL: On exponential bases for the Sobolev spaces over an interval. J. Math. Anal. Appl. 87, 528-550 (1982)

10. Bilalov, BT: On basicity of the system of exponents, cosines and sines in $L_{p}$. Dokl. Akad. Nauk, Ross. Akad. Nauk 365(1), 7-8 (1999)

11. Bilalov, BT: On basicity of some system of exponents, cosines and sines in $L_{p}$. Dokl. Akad. Nauk, Ross. Akad. Nauk 379(2), 7-9 (2001)

12. Huseynli, AA: On the stability of basisness in $L_{p}(1<p<\infty)$ of cosines and sines. Turk. J. Math. 35, 47-54 (2011)

13. Cruz-Uribe, DV, Fiorenza, A: Variable Lebesgue Spaces: Foundations and Harmonic Analysis. Springer, Berlin (2013)

14. Kovacik, O, Rakosnik, J: On spaces $L^{p(\cdot)}$ and $W^{k, p(\cdot)}$. Czechoslov. Math. J. 41(116), 592-618 (1991)

15. Xianling, F, Dun, Z: On the spaces $L^{p(x)}(\Omega)$ and $W^{m, p(\cdot)}(\Omega)$. J. Math. Anal. Appl. 263, 424-446 (2001)

16. Bilalov, BT, Guseynov, ZG: $\mathscr{K}$-Bessel and $\mathscr{K}$-Hilbert systems and $\mathscr{K}$-bases. Dokl. Math. 80(3), 826-828 (2009)

17. Muradov, TR: On bases from perturbed system of exponents in Lebesgue spaces with variable summability exponent. J. Inequal. Appl. 2014, 495 (2014)

18. Levin, BY: Distribution of the Root of Entire Functions. GITTL, Moscow (1956) 632 pp.

19. Il'in, VA: Necessary and sufficient conditions of basicity in and equiconvergence with trigonometric series of spectral expansions and expansions in the systems of exponents. Dokl. Akad. Nauk SSSR 273, 789-793 (1983)

20. Marchenko, VA: Spectral Theory of the Sturm-Liouville Problem. Naukova Dumka, Kiev (1972) 